\title{
OROBANCHE L. (OROBANCHACEAE) SECT. TRIONYCHON WALLR., EN ANDALUCÍA
}

\author{
Antonio J. PUJADAS SALVÀ
}

RESUMEN. Orobanche L. (Orobanchaceae) sect. Trionychon Wallr., en Andalucia. Se ha llevado a cabo la revisión de las especies de la sección Trionychon Wallr. del género Orobanche en Andalucía, sur de la Península Ibérica. Para ello se ha realizado una exhaustiva prospección botánica y una revisión crítica de la información bibliográfica existente y de los principales herbarios nacionales. Para las siete especies identificadas aportamos su corología, características ecológicas y hospedantes. Resaltamos la presencia de especies de marcado interés corológico como son $O$. lavandulacea Rchb. y $O$. purpurea Jacq. como taxones no citados en gran parte del territorio.

Palabras clave. Orobanche, jopo, parásito, corología, Andalucía.

ABSTRACT. Orobanche L. (Orobanchaceae) sect. Trionychon Wallr., in Andalusia. A botanical survey has been carried out in order to review the Sect. Trionychon Wallr. of the genus Orobanche L. in Andalusia, south of the Iberian Peninsula. For this, an exhaustive botanical exploration and a critical review of the existing bibliographical information and of the principal national herbaria has been accomplished. For the seven identified species we provide its chorology, ecological characteristics and hosts. We emphasize the presence of taxa of important chorological interest as O. lavandulacea Rchb. and $O$. purpurea Jacq. as species not cited in a large part of the territory.

Key words. Orobanche, broomrape, parasite, chorology, Andalusia.

\section{INTRODUCCIÓN}

No hay ninguna revisión del género Orobanche para Andalucía, por lo que, la ya clásica obra de Willkomm (1870 \& 1893) sigue siendo una referencia obligada para su estudio. Sin embargo, para Portugal, existe un tratado para la familia Orobanchaceae (Guimaraes, 1903) de gran valor al tratarse de un territorio limítrofe con el nuestro. Para nuestro ámbito de estudio se han efectuado algunas aportaciones florísticas (Pujadas Salvà et al. 1994 y Molino-Olmedo et al. 1995) que suponen el primer avance para su conocimiento. Contamos, además, con diversas floras y catálogos florísticos como los de Pérez Lara (1889), Cuatrecasas (1929), Galiano \& Heywood (1960), Fernández López (1983), Valdés et al. (1987), Sagredo (1987); Molero Mesa \& Pérez Raya (1987) y Fernández et al., (1991), pero que en general son incompletos o a veces insatisfactorios con el tratamiento taxonómico que se da al género. Como antecedente a este trabajo recientemente hemos publicado una aportación a su conocimiento para la provincia de Almería (Pujadas Salvà \& 
Lora González,1996).

Al tratarse de un género conflictivo, cuyas especies son, a veces, de difícil determinación $y$, en general, escasamente representado en los herbarios, consideramos necesaria esta contribución a su conocimiento para el sur de la Península Ibérica, conscientes además de que no se trata de un trabajo definitivo y que estudios posteriores van a permitir su mejor conocimiento tanto taxonómico como corológico.

Aportamos las descripciones morfológicas de las especies de Orobanche Sect. Trionychon localizadas en el territorio, además de unas claves de determinación tanto para material fresco como para material de herbario. Se indican también para cada una de ellas, las citas bibliográficas correspondientes a iconos de calidad publicados en obras fácilmente accesibles. Aportamos un icono original, realizado sobre material andaluz, correspondiente a $O$. lavandulacea Rchb. ya que los que conocemos son incompletos o de difícil localización. Se indican las citas correspondientes al material estudiado y los posibles hospedantes conocidos para cada una de las especies.

\section{MATERIAL Y MÉTODO}

Se ha llevado a cabo una revisión bibliográfica de los trabajos florísticos sobre el territorio de estudio, tomando como referencias básicas los trabajos de Willkomm (1870; 1893), Beck $(1890 ; 1930)$ y Chater \& Webb (1972). Igualmente se ha realizado una revisión crítica, total o parcial, de los especímenes conservados en los herbarios $\mathrm{ABH}, \mathrm{ALME}, \mathrm{BC}, \mathrm{BCC}, \mathrm{BCF}, \mathrm{COA}, \mathrm{COFC}$, COLEGIO LA SALLE DE ALMERIA, GDA, GDAC, JACA, JAEN, MA, MAF, MGC, MPU, MUB, RNG y SEV.

Además, se ha llevado a cabo una prospección botánica en toda la región para la recolección de material vivo a fin de lograr una mayor precisión en la identificación y descripción de las especies, así como para la determinación de sus correspondientes hospedantes. Los testimonios de estas recolecciones se conservan en el Herbario COA del Departamento de Ciencias y Recursos Agrícolas y Forestales, de la Universidad de Córdoba.

Se citan sólo los hospedantes que hemos podido contrastar como seguros, para cada especie de Orobanche, por lo que no se han tenido en cuenta algunas referencias de las etiquetas de herbario que consideramos como poco fiables.

Para la identificación y nomenclatura de los diferentes taxones hemos seguido, para las Orobanchaceae, principalmente a Beck (1890; 1930) y Greuter et al. (1989) y para el resto de las especies a Tutin et al. (1964-1980), Valdés et al. (1987) y Talavera et al.(1999). Hemos seguido las recomendaciones de Brummit \& Powell (1992) al abreviar los nombres de los autores botánicos

\section{RESULTADOS}

El género Orobanche está integrado por las secciones Trionychon Wallr. y Orobanche L., que constituyen dos grupos taxonómicos bien delimitados, sus caracteres diferenciales más significativos son:

-Tallos simples o ramificados. Flores sésiles o pediceladas, con dos bractéolas. Cáliz con los segmentos unidos, campanulado. Corola azul o púrpura, a veces blanquecina. Anteras blancas. Estigma blanco o ligeramente teñido de azul o púrpura

\section{sect. Trionychon}

-Tallos simples. Flores sésiles, sin bractéolas. Cáliz dividido en dos segmentos laterales, a veces unidos abaxialmente. Corola blanca, amarilla, marrón, ametistea o roja, raramente azul o púrpura. Anteras amarillas, pardas o 
grisáceas, raramente blancas. Estigma amarillo, naranja, rojo o púrpura, raramente blanco

\section{sect. Orobanche}

Se han identificado siete especies diferentes de Orobanche para la Sect. Trionychon, cuya relación, descripción sobre material andaluz, huéspedes y citas de herbario se comentan a continuación.

\section{Clave de la Sect. Trionychon}

\section{a) material fresco}

1. Cáliz (13)16-21 mm. Corola de (18)21-30 mm, azul violeta intenso, blanquecina en la base .....

6. $O$. arenaria

Cáliz 4-16 mm. Corola de 12-21 mm, blanquecina en la base, blanquecina a azul intenso en el ápice

2. Cáliz (10)13-16 mm, con dientes $1,5-3$ veces la longitud del tubo 5. O. schultzii Cáliz 4-12 mm, con dientes igualando o menores que la longitud del tubo 3

3. Planta de $8-20 \mathrm{~cm}$. Inflorescencia pauciflora. Dientes del cáliz lanceolados con ápice acuminado, filiforme. Corola azul brillante en el ápice. Anteras glabras 2. O. nana Planta de (7) $12-31 \mathrm{~cm}$. Inflorescencias multifloras. Dientes del cáliz subulados, lanceolados o triangulares. Corola blanquecina o azul en el ápice. Anteras glabras o pelosas . 4

4. Tallo ramificado 5

Tallo simple 7

5. Cáliz 4-6 mm. Corola 12-15(17) mm, blanquecina o azul pálido en el ápice. Parásita de plantas cultivadas 1. O. ramosa Cáliz 7-11 mm. Corola (15)17-21 mm, azul pálida a azul oscuro. Parásita de plantas silvestres ... 6

6. Planta de (7)12-20 mm, blanquecino. Inflorescencia $(2,5) 5-8 \mathrm{~cm}$. Cáliz con dientes igualando el tubo, blanquecino 3. O. mutelii Planta de (25)33-53 cm, púrpura oscuro.
Inflorescencia (12)19-47 cm. Cáliz con dientes menores que el tubo, azul oscuro

4. O. lavandulacea

7. Planta (7) $12-20 \mathrm{~cm}$. Cáliz con dientes igualando el tubo. Corola de azul pálido a azul brillante en el ápice. 3. O. mutelii Planta 19-29 cm. Cáliz con dientes mucho menores que el tubo. Corola con las venas marcadas, teñidas de púrpura, a veces de azul pálido 7. O. purpurea

\section{b) material de herbario}

1. Dientes del cáliz 1,5-3 veces la longitud del tubo 5. O. schultzii Dientes del cáliz igualando o menores que la longitud del tubo. 2

2. Cáliz (13)16-21 mm. Corola de (18)21-30 mm. 6. O. arenaria Cáliz menor de $12 \mathrm{~mm}$. Corola menor de $21 \mathrm{~mm}$ 3

3. Cáliz 4-7,5 mm. Corola 12-16(17) mm ............. 4 Cáliz 7-12 mm. Corola (15) 17-22 mm ............. 5

4. Planta $17-35 \mathrm{~cm}$, muy ramificada desde la base Inflorescencia 10-25 cm, laxa. Cáliz 4-6 mm, dientes triangulares ........................ 1. O. ramosa Planta $8-20 \mathrm{~cm}$, simple o ramificada. Inflorescencia 3,5-7(8) cm, densa, algo laxa en la base. Cáliz 5-7,5 mm, dientes lanceolados con ápice acuminado filiforme 2. O. nana

5. Planta (7) $12-20 \mathrm{~cm}$. Cáliz con dientes igualando el tubo 3. O. mutelii Planta $19-53 \mathrm{~cm}$. Cáliz con dientes menores que el tubo. 6

6. Planta $19-29 \mathrm{~cm}$. Tallo simple ... 7. O. purpurea Planta (25)33-53 cm. Tallo ramificado... 4. O. lavandulacea

\section{Diagnosis y catálogo florístico}

\section{Sect. TRIONYCHON Wallr.}

Tallo pubescente glandular. Flores sésiles 
o a veces las inferiores cortamente pediceladas, raramente con pedicelos largos, con dos bractéolas. Cáliz con los segmentos unidos, campanulado, profunda y regularmente tetradentado, con dientes dispuestos por pares lateralmente, a veces con un quinto diente de menor tamaño situado adaxialmente. Corola con el labio superior bilobado, el inferior trilobado con los lóbulos iguales o subiguales, pubescente glandular, azul o púrpura, a veces blanquecina. Anteras blancas. Estigma blanco o ligeramente teñido de azul o púrpura.

1. Orobanche ramosa L., Sp. Pl. 633 (1753) Phelypaea ramosa (L.) C.A. Mey.

Ic.: Beck (1930: 62, fig. A); Bonnier (1911-1935: pl. 463, fig. 2144 [sub Phelypaea ramosa]); Rumsey \& Jury (1991: 275, fig. 1A; 278, fig. 4-A).

Planta de 17-35 cm. Tallo de 1,2-3 mm de diámetro en su mitad, escasamente engrosado en la base (3-12 $\mathrm{mm}$ de diámetro), muy ramificado desde la base, pubescente glandular, blanquecino. Hojas de 3-7 x 1,5-3 mm, ovadas a lanceoladas, esparcidas. Inflorescencia de 10-25 x 2-2,5 cm, multiflora, laxa, cilíndrica, con ápice cónico. Flores sésiles, las basales frecuentemente con pedicelos de hasta $4 \mathrm{~mm}$. Brácteas de 4-6 x 1-2 mm, ovadas o ampliamente lanceoladas, blanquecinas. Bractéolas de 3-4,5 x 0,4-0,7 mm, linear lanceoladas, blanquecinas. Cáliz de 4-6 mm, con dientes triangulares, igualando su tubo, blanquecino. Corola de 12-15(17) mm, erecto patente, hinchada en la base, poco estrecha en la inserción de los filamentos, de 2-2,6 mm de ancho y tubular infundibuliforme en el ápice, de 4-6 mm de ancho en la garganta, pubescente glandular, blanquecina o azul pálido en el ápice. Labio superior emarginado con lóbulos porrectos, el inferior con lóbulos ovados, obtusos, porrectos, de margen cortamente ciliado, con pelos glandulares de 0,2-0,3 mm. Filamentos insertos horizontalmente a (3)4-5 $\mathrm{mm}$ de la base de la corola, glabros o subglabros (pelos visibles sólo al microscopio estereoscópico); anteras glabras. Ovario glabro. Estilo esparcidamente peloso glandular, pelos algo más densos hacia el ápice, blanco. Estigma blanco o violeta pálido.

Sobre plantas cultivadas, principalmente solanáceas (Lycopersicum esculentum Mill. y Nicotiana tabacum L.) y citada también en los cultivos tradicionales de Cannabis sativa L., en el piso termomediterráneo. 50-800 m. (IV)VI-VIII(IX).

\section{Material estudiado:}

ALMERIA: Presa Níjar, 2-VI-62, R. Sagredo, $\mathrm{H}^{\circ}$ LA SALLE $s / n$.

GRANADA: Almuñécar, Torre del Cambrón, in agro argilloso-glareoso, matrix Solanum lycopersicum, 28-IV-1952, H. Roivainen, BC 143760. La Zubia, sobre tabaco, 27-V-1988, J. Oliveira, COA 13816. Purchil, sobre tabaco, 25VII-1988, J. Oliveira, COA 13809. Mercagranada, sobre tabaco, 25-VII-1988, J. Oliveira, COA 13815. Santa Fé, sobre tabaco, 25-VII-1988, J. Oliveira, COA 13817. Pinos Puente, sobre tabaco, 25.VII1988, J. Oliveira, COA 13798. Trasmulas, sobre tabaco, 26-VII-1988, J. Oliveira, COA 13820. Aeropuerto de Granada, sobre tabaco, 26-VII-1988, J. Oliveira, COA 13856. Armilla, sobre tabaco, 27VII-1988, J. Oliveira, COA 13855. Ambros, sobre tabaco, 25-VIII-1988, J. Oliveira, COA 13810. Belicena, sobre tabaco, 25-VIII-1988, J. Oliveira, COA 13813. El Jau, sobre tabaco, 25-VIII-1988, J. Oliveira, COA 13851. Churriana de la Vega, sobre tabaco, 25-VIII-1988, J. Oliveira, COA 13852. San Fernando, sobre tabaco, 25-VIII-1988, J. Oliveira, COA 13800. Bobadilla, sobre tabaco, 25-VIII-1988, J. Oliveira, COA 13801. La Gabia, Vega de Granada, sobre tabaco, 14-X-1988, J. Oliveira, COA 13551. Cullar de Vega, sobre tabaco, 5-X-1989, A. Pujadas \& J. Oliveira. COA 13605. Chauchina, sobre tabaco, 5-X-1989, A. Pujadas, COA 13837.

Nota: Willkomm (1870: 629) recoge además algunas citas para Cádiz, Granada y Gibraltar, mientras que Beck (1930: 68) sólo la reconoce para las provincias del norte de la Península Ibérica. Algunos autores como Pérez Lara 
(1889: 141) y Valdés (1987: 557) incluyen en este taxon a $O$. nana y $O$. mutelii.

2. Orobanche nana (Reut.) Beck, Biblioth. Bot. 19: 91 (1890)

Orobanche ramosa L. subsp. nana (Reut.) Cout.

Phelypaea nana (Reut.) Rchb. f.

Phelypaea mutelii (F.W. Schultz) Reut. var. nana Reut.

Ic.: Coste (1937: 63, fig. 2805 [sub Phelypaea nana]); Kreutz (1995: 46, 47 [sub Orobanche ramosa]); Valdés (1987: 557 [sub Orobanche ramosa]).

Planta de 8-20 cm. Tallo de (1)1,5-3,5 $\mathrm{mm}$ de diámetro en su mitad, algo engrosado 0 bulboso en la base [4-9(12) $\mathrm{mm}$ de diámetro], simple o ramificado, pubescente glandular, blanquecino o azulado. Hojas de (3)5-9(12) x 2-3 mm, ovadas, raramente lanceoladas, esparcidas. Inflorescencia de 3,5-7(8) x 2-3 $\mathrm{cm}$, cilíndrica, ápice redondeado, generalmente densa, algo laxa en la base. Flores sésiles o a veces, en la base de la inflorescencia, cortamente pediceladas, con pedicelos hasta 4 mm. Brácteas de 5-8 x 2,5-3,5 mm, ampliamente lanceoladas, blancas a veces teñidas de azul. Bractéolas 5-7 x 0,6-0,9(1,1) mm, linear lanceoladas a estrechamente lanceoladas, blancas a veces teñidas de azul. Cáliz de 5-7,5 $\mathrm{mm}$, con dientes lanceolados con ápices acuminados filiformes, mayores que la longitud del tubo, blanco a veces azul oscuro. Corola de 12-16(17) $\mathrm{mm}$, de erecto patente a patente, ligeramente hinchada en la base, genuflexa, estrecha en la inserción de los filamentos, de (1) 1,5-2 $\mathrm{mm}$ de ancho, y tubular infundibuliforme en el ápice, de 4-5 mm de ancho en la garganta, pubescente glandular, blanquecina en la base, azul brillante en el ápice, a veces intensamente azul oscuro. Labio superior de la corola profundamente emarginado con los lóbulos patentes o porrectos, el inferior con lóbulos elípticos, subobtusos, porrectos a veces algo deflexos, de margen cortamente ciliado, con pelos glandulares de 0,1-0,2(0,3) mm. Filamentos insertos horizontalmente a 4-6 $\mathrm{mm}$ de la base de la corola, pubérulos, raramente subglabros en la parte inferior y pelos glandulares sésiles bajo las anteras; anteras glabras, raramente subglabras (con 1-2 pelos de 0,3-0,5 mm). Ovario glabro. Estilo glabro o subglabro, blanco, a veces purpúreo. Estigma blanco.

En herbazales subnitrófilos del piso termo y mesomediterráneo, sobre Aetheorhiza bulbosa (L.) Coss., Coleostephus myconis (L.) Rchb., Hedypnois cretica (L.) Dum. Cours., Malva hispanica L., Sherardia arvensis L., Sonchus oleraceus L., Trifolium campestre Schreb. 0-1600 m. III-V.

\section{Material estudiado:}

ALMERIA: Vivero de los Alamicos, 30SWG6772, 1210 m, 24-V-1988, Blanca, Cueto, Rebollar \& Robles, ALME s/n. Barranco del Caballar, sobre Galium verrucosum (sic.), s/f, $R$. Sagredo, $\mathrm{H}^{\circ} \mathrm{LA}$ SALLE s/n. Níjar, El Hoyazo, sobre Artemisia, s/f, R. Sagredo, $\mathrm{H}^{\mathrm{o}} \mathrm{LA}$ SALLE s/n. Sierra de Filabres, $1100 \mathrm{~m}, 1-\mathrm{V}-1958$, R. Sagredo, $\mathrm{H}^{\circ}$ LA SALLE s/n. Barranco Palmer, 24-III-1960, R. Sagredo, $\mathrm{H}^{\circ}$ LA SALLE s/n. Barranco Palmer, sobre Crepis bulbosa, 29-III-60, R. Sagredo, $\mathrm{H}^{\circ}$ LA SALLE s/n. Barranco Palmer, sobre Aetheorhiza bulbosa, 13-IV-1960, $R$. Sagredo, $\mathrm{H}^{\circ}$ LA SALLE s/n. El Romeral, 16-V1962, R. Sagredo, $\mathrm{H}^{\circ}$ LA SALLE s/n. Punta del Sabinar, arenas, 15-IV-1984, B. Díez Garretas \& A. Asensi, MGC 17954.

CÁDIZ: Finca El Zapatero, Benalup, Parque Los Alcornocales, 4-V-1995, A. Pujadas \& A. Lora, COA 17457. Sanlúcar de Barrameda, Monte de Algaida, casa del guarda, 25-V-1996, N. Bernal, COA 13746. Grazalema, Paseo de las Encinas y Laureles, 28-IV-1989, V.E. Martín Osorio, MGC 33145. Vejer de la Frontera, 14-IV-1956, D.M.C. Brinton-Lee, SEV 82122. San Roque, El Almendral, parasitic on Gazania, 19-IV-1959, D.M.C. BrintonLee, SEV 82123. Sa de Líjar, Algodonales, 500$1000 \mathrm{~m}$, calizas jurásicas, 16-V-1980, A. Aparicio, SEV 115472.

CÓRDOBA: Córdoba, Medina Azahara, 1-V-1986, A. Pujadas, COA 13799. Próx. Medina Azahara, 
sobre Hedypnois cretica (L.) Dum.-Courset, 1-V1986, A. Pujadas et al., COA 13836. Hornachuelos, E.A. cerca de la nueva presa del Tinte, 19-III-1987, F. Navarro \& J.O. Velloso, COA 13802. Cerca del río Guadalmellato, 10-V-1987, A. Pujadas \& J. Recassens, COA 28175. Hornachuelos, cerca de Navalahiguera, Dehesa El Asiento, 24-IV-1988, E. Navarro, COA 13518. Alcolea, cerca del Pantano Guadalmellato, sobre Coleostephus myconis (L.) Reichenb., 12-V-1988, A. Pujadas, COA 13609. Alcolea. Km 5 al N de Alcolea, sobre Sherardia arvensis L. 12-V-1988, J.O. Velloso \& A. Pujadas, COA 13812. Alcolea, Pantano Guadalmellato, sobre Trifolium campestre Schreber, 12-V-1988, J.O. Velloso \& A. Pujadas, COA 13834. Alcolea, Pantano Guadalmellato sobre Sonchus oleraceus L., 12-V1988, J.O. Velloso \& A. Pujadas, COA 13835. Km 5 al Norte de Alcolea, sobre Malva hispanica L., 21-V-1988, J.O. Velloso \& A. Pujadas, COA 13811. Presa San Rafael, cerca del Guadalmellato, 5-V1990, A. Pujadas \& P. Poyato, COA 17458. Córdoba, Fuente del Orquín, junto Los Morales 17-IV-1991, J. López, COA 17456. Km 18 ctra. CórdobaVillaviciosa, 2-IV-1995, M.J. Retamosa, COA 13870. Cerca de la «Cueva de Los Murciélagos», Zuheros, 15-IV-1995, R. Amores, COA 17634. Doña Mencía, entre Doña Mencía y Zuheros, UG84, 600 m, 14-V-1978, Muñoz, COFC 5959. Villaviciosa de Córdoba, Arroyo posterior al Arroyo Pajaroncillo, 18-V-1980, Corral \& Fernández, COFC 2599 y SEV 118666. Valle del Guadalmellato, Cerro Estribo, dcha. de la presa del Embalse, 25-IV-1980, M. Arenas \& E. Domínguez, COFC 6543. Camino a la dcha. de la carretera Hornachuelos-San Calixto, en dirección a la finca "Los Rayos Altos", 1-VI1981, Fernández \& Porras, COFC 6545.

GIBRALTAR: Catalan Bay, arenas mezcladas con rocas calizas, 16-V-1985, J. Bensusan et al., SEV 124874.

GRANADA: Sierra de Alhama, Ventas de Zafarraya, 3-V-1986, J.E. Hernández \& M. Clemente, COA 17453. Cortijo Los Charcos, Baza, 15-V-1990, E. Martinez, COA 17459. Sierra de Parapanda, 1600 m, 28-VI-2000, A. Pujadas, L. Plaza \& B. Román, COA 29509. Dehesa de Alfacar, 17-V-1908, J.L. Diez Tortosa, GDA s/n.

HUELVA: Isla Cristina, playa del Hoyo, PB 51, 5 m, 14-IV-1996, Camuñas, Juan, Serra, Cristóbal \& Crespo, ABH 30955. Aroche, cortijo de Maribarbe, 30SPC8306, 26-III-1998, M. López, COA 25543. Hinojales-Fuentes de León, cerro del Guijo, en un olivar sobre mármoles calcodolomíticos, 22-IV1998, J.M. Delgado, COA 25308. El Terrón, Lepe, en cardunal sobre Asteráceas, 29SPB6121, 15-VI1998, E. Sánchez Gullón, COA 28432. Entre Hinojos y Almonte, pinares de Pinus pinea, 11-III-1977, B. cabezudo \& S. Silvestre, SEV 110049. Sa de Aracena,
25-IV-1978, J. Rivera, MGC 8816. S ${ }^{\text {a de Aracena, }}$ Aracena, cordillera, 25-IV-1978, J. Rivera, SEV 48492

JAÉN: Despeñaperros, límite de la provincia de Ciudad Real, 30SVH5651, $700 \mathrm{~m}, 1-\mathrm{V}-1977, P$. Montserrat, JACA 27477 (sub O. lavandulacea in Fernández López \& Montserrat Recoder, 1985: 84). MÁLAGA: Campillos, Sierra de la Pizarra, cerro Pardo, fuente del Carrizo, 30SUF4090, 9-III-1997, A. Lora, L. Plaza \& J.C. Moreno, COA 24848. Sierra Llana, margen de la pista, 30SUF4190, 500 m, 26-III-1998, A. Pujadas, L. Plaza \& R. Rubio, COA 25556. Playa término de Estepona, UF03, 13 III-1978, B. Díez, MGC 4829. Sa Bermeja, Estepona, UF03, 8-IV-1978, A. Asensi \& B. Díez, MGC 4943. Entre Alhaurín de la Torre y Alhaurín el Grande, UF 55, 9-IV-1978, A. Asensi \& B. Diez, MGC 4942. S Tejeda, Tajo de la Campana, VF08, 12-IV-1980, J.M. Nieto, MGC 7501. Caracolillos, UF55, 13-IV1980, Salvo \& Conde, MGC 6510. Sa de Nerja, Carril de la Fuente del Esparto, VF27, 26-IV-1982, M. Trigo, MGC 10953. Sa Blanca, Ventorro la Chata, 7-V-1982, Merino \& Guerra, MGC 11781.

SEVILLA: El Real de la Jara, Finca El Pimpollar, 30SQC5605, 19-V-1997, J.M. Delgado. COA 23756. Sierra del Pimpollar, Cortijo de las Aguilas, 30SQC5605, 9-VII-1997, M. López, COA 23755. Oromana, Alcalá de Guadaira, en protosuelo básico sobre nanocaméfitos y terófitos, 28-III-1998, COA 28427. Pinares de Bollullos, 19-III-1966, E.F. Galiano \& J. Novo, E. Sánchez Gullón SEV 129292.

3. Orobanche mutelii F.W. Schultz in Mutel, Fl. Franç. 2:353 (1835)

O. ramosa L. subsp. mutelii (F.W. Schultz) Cout.

Phelypaea caesia sensu Willk., non Rchb. Phelypaea mutelii (F.W. Schultz) Reut.

Ic.: Bonnier (1911-1935: pl. 463, fig. 2144b [sub Phelypaea mutelii]); Coste (1937: 64, fig. 2806 [sub Phelypaea mutelii]).

Planta de (7)12-20 cm. Tallo de $(2,5) 4-$ 7(10) mm de diámetro en su mitad, escasamente engrosado en la base (8-13 mm de diámetro), simple o ramificado, pubescente glandular, blanquecino. Hojas de 6-14 x (1,5)3-6 mm, ovadas o lanceoladas. Inflorescencia de $(2,5) 5$ $8 \times 2,5-3,7 \mathrm{~cm}$, densa, ápice redondeado. Flores 
sésiles o las inferiores escasamente pediceladas, a veces con pedicelos de hasta 4 $\mathrm{mm}$. Brácteas de 6-13 x 2,5-5 mm, ovadas a lanceoladas, blanquecinas. Bractéolas de 5-10 x 0,7-1 mm, linear lanceoladas, blanquecinas. Cáliz de 7-10 mm, con dientes subulados, aproximadamente de la misma longitud que el tubo, raramente de ápice filiforme, blanquecino. Corola de (15)18-22 mm, de erecta a erecto patente, a veces patente, recta 0 ligeramente curvada, raramente genuflexa en la madurez, hinchada en la base, estrecha en la inserción de los filamentos, de 1,5-2(3) $\mathrm{mm}$ de ancho, e infundibuliforme en el ápice, de 56(8) $\mathrm{mm}$ de ancho en la garganta, pubescente glandular, blanquecina en la base, de azul pálido a brillante en el ápice. Labio superior emarginado, con lóbulos cortos, porrectos o patentes, el inferior trilobado con lóbulos iguales o subiguales, ovados de ápice redondeado, porrectos, de margen cortamente ciliados con pelos glandulares de 0,1-0,3 mm. Filamentos insertos horizontalmente a (3)46(7) $\mathrm{mm}$ de la base de la corola, pubérulos en la parte inferior; con pelos glandulares sésiles por debajo de las anteras; anteras glabras o con 1-6 pelos, de hasta $0,5 \mathrm{~mm}$, en la base. Ovario glabro o subglabro con escasos pelos en el ápice. Estilo glabro o con pelos glandulares esparcidos, blanco. Estigma blanco o azul pálido.

En matorrales xerofíticos degradados de los pisos termo al supramediterráneo, sobre Artemisia glutinosa DC. y Leontodon longirostris (Finch \& P.D. Sell) Talavera. 202000 m. III-VII.

\section{Material estudiado:}

ALMERIA: Cortijo de Gógora, sobre Sonchus y Launaea, 19-III-1943, Hno. Jerónimo, ALME 1720. Al pie de Sierra Cabrera, sobre leguminosa, 22-IV1954, Hno. Jerónimo, ALME 1725. Venta de Yesos (entre Tabernas y Sorbas), litosuelo de yesos del Mioceno Superior, 30SWG60, 500-550m, 7-V-1983, R. Lázaro Suau, ALME s/n. Pico María, Sierra María, 30SWG7170, 2-VII-1987, Blanca \& Cueto,
ALME s/n. Cerca de los Cuernos, Sierra María 30SWG6465, 1500 m, 8-VI-1988, M. Cueto, ALME $\mathrm{s} / \mathrm{n}$. Cerca de la Ermita de la Virgen de la Cábeza, Sierra María, 30SWG7271, 20-VI-1988, M. Cueto, ALME s/n. Arroyo del Puntal del Moral, Sierra María, 30SWG7071, 1400 m, 22-VI-1988, M. Cueto, ALME s/n. Sierra Almagro, 30SWG9936, 11-V1990, E. Martínez, COA 174499. Playa de Los Muertos, Carboneras, 30SWF9890, 20 m, 13-IV1994, A. Pujadas \& P. Poyato, COA 17450. Puertecico, Sta. María de Nieva, sobre Artemisia glutinosa, 30SWG8953, $850 \mathrm{~m}, 11-\mathrm{V}-1994$, A. Pujadas, COA 17451. Base de Sierra Cabrera, playa del Castillejo de Mocenas, entre albaidal, 30SXG0204, 5-IV-1990, J. Peñas \& $M^{a}$.J. Salinas, GDAC 33375. Sierra María, sobre Thymus, s/f, $R$. Sagredo, $\mathrm{H}^{\circ}$ LA SALLE s/n. Venta de los Castaños, s/f, $R$. Sagredo, $\mathrm{H}^{\circ}$ LA SALLE s/n. Barranco Palmer, sobre Picridium (sic.), 18-III-1959, R. Sagredo, $\mathrm{H}^{\circ}$ LA SALLE $\mathrm{s} / \mathrm{n}$. Barranco S. Indalecio, 6-IV-1959, R. Sagredo, $\mathrm{H}^{\circ}$ LA SALLE s/n. Sierra de María, 27VI-1962, R. Sagredo, $\mathrm{H}^{\circ}$ LA SALLE s/n. Barranco Palmer, sobre Leontodon taraxacoides subsp. longirostris, 13-IV-1966, R. Sagredo, $\mathrm{H}^{\circ}$ LA SALLE s/n. Roquetas, 3-IV-1969, R. Sagredo, Ho LA SALLE $\mathrm{s} / \mathrm{n}$. Hortichuelas Bajas, 19-IV-1973, R. Sagredo, $\mathrm{H}^{\circ}$ LA SALLE s/n. Sierra Alhamilla, 30SWF49, 5IV-1983, Lázaro Suau, MGC 13410. Pechina, Ramblas, 9-IV-1979, J.A. Devesa et al., SEV 51345. CÁDIZ: Puerto Santa María - Jerez, Sa de San Cristóbal, 29SOA5056, $60 \mathrm{~m}, 21-\mathrm{IV}-1970, P$. Montserrat, JACA 42170. Digues des chemins entre $S^{a}$ María et Jerez, près Cadix, 9-IV-1873, $R$. Fritze, MPU $s / n$.

CÓRDOBA: Entre Palenciana y Benamejí, Cortijo Segovia, margen izquierda del Genil, 21-V-1981, $F$. Infante \& E. Hernández, COFC s/n.

GIBRALTAR: W side of rock, upper platform of cable car. c $500 \mathrm{ft}$, rocky walls, 24-III-1969, V.H. Heywood, D.M. Moore et al., RNG 22.

GRANADA Fuentenueva-Venta Micena, sobre Vella pseudocytisus L. (sic.), entre campo de garbanzocebada, 24-V-1996, P. Prados \& J.L. Vivero, COA 22106. Venta Micena, junto campos de cultivo, 30SWG57, 7-VI-1996, J.L. Vivero, COA 13681. La Sagra, 21-V-1997, P. Luque, COA 13563. Sierra Nevada, Nigüelas, pista al Barranco Alisos, 22-VII1997, P. Prados, T. Parras \& R. Jiménez, COA 13550. Almuñécar, La Herradura, Punta de la Mona, 20-IV-1988, E. Arrojo Agudo, GDAC 33296. Laroles, VF9995, $975 \mathrm{~m}$, en un barranco muy húmedo parasitando a Oxalis (sic.), 22-IV-1988, A. Hervás, GDAC 28830. Iznalloz, $S^{\text {a }}$ del Rayo, subida al Alto del Muerto, 30SVG4641, parásita sobre compuestas, 20-V-1989, F.J. García \& L. Gutiérrez, GDAC 37114. Sa de La Sagra, amongst stones, calcareous soil and screes, WG3699, 1850-2300 m, 
Valdés et al., SEV s/n.

JAÉN: Sierra de Cazorla, Poyos de la Carilarga, 18V-1997, P. Luque, COA 13697. Sierra Mágina, El Almadén, 4-VII-1998, A. Pujadas \& L. Plaza, COA 25555. S Mágina, Mágina Occidental, 1800 m, 4VII-1926, Cuatrecasas, BC 46560. La Cerradura, 800 m, 16-IV-1988, G. Blanca, GDAC 27604. S Seca, El Chaparral, calcareous soils, WH2800, 1800 m, 29-VI-1988, B. Valdés et al., SEV s/n.

MÁLAGA: Montes de Málaga, s/f, J.M. Cazorla, COA 17454. Torcal de Antequera, 7-VI-1976, S. Guerra, GDAC 4442. Playa del Campamento Benítez, UF65, 1-IV-1977, M.A. Zaragoza, MGC 5550. Rincón de la Victoria, UF8164, prado, 27-IV1984, S. Pérez \& Hidalgo, MGC 14010. S ${ }^{\mathrm{a}} \mathrm{de}$ Torremolinos, UF65, 29-IV-1984, S. Pérez Sanz, MGC 16531 y MGC 16530. $S^{a}$ de Mijas, Llanos de la Plata, 13-III-1987, Ternero \& Gil, MGC 22212. Ronda, Cortijo de Planilla, UFO $866,730 \mathrm{~m}$, dolomías, 29-III-1988, D. Montilla, MGC s/n. Marbella, Punta Ladrones, 17-III-1990, UF4339, A. Márquez et al., MGC 331020. Antequera, Torcal, amongst calcareous rocks and stones, $1150-1300 \mathrm{~m}$, 14-VI-1988, B. Valdés et al., n ${ }^{\circ} 105 / 88$, SEV 135668 (sub O. ramosa in Valdés \& Talavera, 1991: 204).

Nota: Beck (1930: 76) la cita además para Sevilla, Jerez, Cádiz, San Roque y Gibraltar. Consideramos que deben de incluirse aquí gran parte de las citas para Andalucía de O. caesia (cf. Pujadas et al., 1997: 281), otras como la de Abu Sbaih \& Jury (1994: 42) para Almería, RNG 1020a, son O. cernua.

\section{Orobanche lavandulacea Rchb., Iconogr.}

Bot. Pl. Crit. 7: 48 (1831)

Phelypaea trichocalyx Webb \& Berthel.

$$
\text { Ic.: Fig } 1 .
$$

Planta de (25)33-53 cm. Tallo de 3-4 mm de diámetro en su mitad, escasamente engrosado en su base (1-1,5 cm de diámetro), ampliamente ramificado, frecuentemente con ramificaciones basales cortas portadoras de yemas estériles, pubescente glandular, púrpura oscuro. Hojas de (8)12-15 x 3-6 mm, de lanceoladas a ovadas. Inflorescencia de (12)1947 x 2,8-3,5 cm, cilíndrica, algo piramidal en su ápice, densa volviéndose laxa en la base. Flores sésiles, las inferiores frecuentemente con pedicelos largos de hasta $13 \mathrm{~mm}$. Brácteas de $9-13$ × 3-5 mm, lanceoladas u ovadas, azul oscuro de base blanquecina. Bractéolas de 7 $10 \times 0,8-1,1 \mathrm{~mm}$, frecuentemente connatas al cáliz, lanceoladas, largamente acuminadas, azul oscuro de base blanquecina. Cáliz de 9-11 mm, con 4(5) dientes subulados, largamente acuminados, menores que el tubo, azul oscuro (marrón oscuro en seco). Corola de 17-21 mm, patente, marcadamente curvada, hinchada en la base, estrecha en la inserción de los filamentos, de 1,5-3 $\mathrm{mm}$ de ancho, tubular y estrechamente infundibuliforme en el ápice, de 4-7 mm de ancho en la garganta, pubescente glandular, azul oscuro intenso, blanca en su base (marrón negruzco en seco). Labio superior profundamente bilobado, el inferior con los lóbulos subiguales, oblongos u obovados, porrectos, obtusos, mucronados, con margen densamente ciliado, con pelos glandulares de 0,2-0,4 mm, azules. Filamentos insertos a 3$5,5 \mathrm{~mm}$ de la base de la corola, glabros 0 subglabros en su base y con escasos pelos glandulares sésiles por debajo de las anteras, blancos; anteras, vilosas en su base con pelos hasta $0,7 \mathrm{~mm}$, raramente glabras. Ovario glabro o subglabro. Estilo subglabro, ligeramente teñido de ametistea. Estigma blanco.

En matorral degradado, subruderalizado, del piso mesomediterráneo. Sobre Bituminaria bituminosa (L.) C.H. Stirt. 400-600 m. V-VI.

\section{Material estudiado:}

CÓRDOBA: Iznájar, cerro del Azucarón, sobre Bituminaria bituminosa, 30SUG8526, $450 \mathrm{~m}, 2-\mathrm{V}$ 1998, A. Pujadas, COA 25304.

JAÉN: Los Cañones Secos, Sierra de Jaén, 1-VI2000, B. Román \& F. Fiestas, COA 29501.

SEVILLA: Constantina, carretera de Constantina a Puebla de los Infantes, 3-VI-1981, P. Escalza et al., SEV 117454.

Nota: Ha sido también citada para la provincia de Jaén por diferentes autores. La referencia 


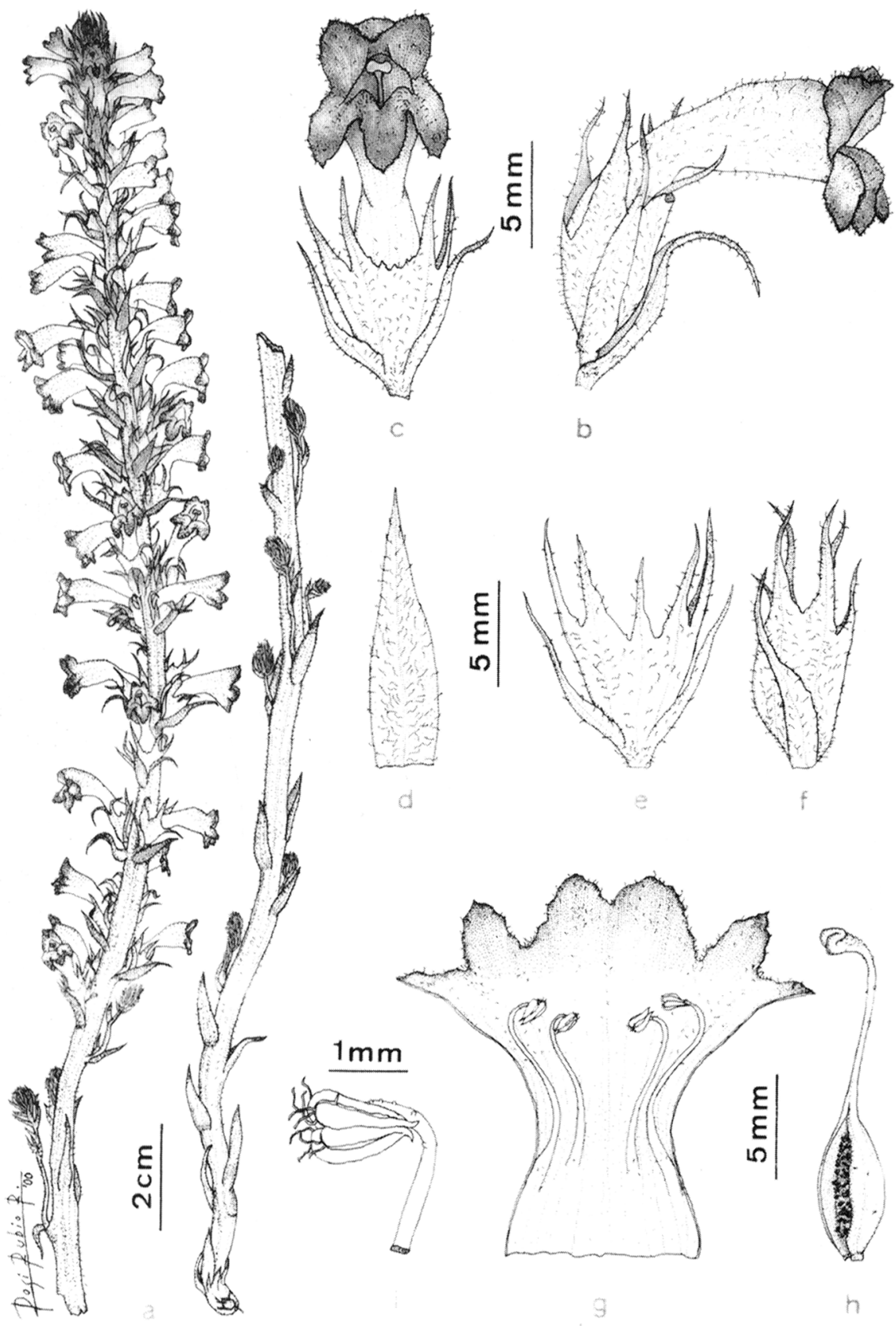

Figura 1. Orobanche lavandulacea. Constantina, carretera de Constantina a Puebla de los Infantes, SEV 117454: a) hábito; b) flor, visión lateral; c) flor, visión frontal; d) bráctea; e) bractéolas y cáliz, visión frontal; f) bractéola y cáliz, visión lateral; g) corola abierta y androceo; h) gineceo; i) antera. Orobanche lavandulacea: a) appearance; b) flower, side view; c) flower, front view; d) bract; e) bracteoles and calyx, front view; f) bracteole and calyx, side view; $g$ ) open corolla and androecium; $h$ ) gynoecium; $i)$ anther. 
de Cuatrecasas (1929: 429) de Rastras de Albánchez, corresponde a $O$. shultzii. La cita de Fernández y Montserrat (1985: 84) corresponde a $O$. nana. No hemos podido encontrar pliegos de testimonio para contrastar las citas de Willkomm (1870: 629) y de Galiano \& Heywood (1960: 157) para esta provincia. Además, Willkomm (1870: 629) la reconoce para la provincia de Málaga. Basándose en estas referencias Beck (1930: 86) sólo indica su presencia en las provincias del sur de España.

Incluimos como su sinónimo a Phelypaea trichocalyx Webb \& Berthel. ya que tanto la descripción original de Webb \& Berthelot (1845: 154) como su icono (Webb \& Berthelot, 1847: tab. 185) coinciden con los caracteres de O. lavandulacea. Este tratamiento es análogo al seguido por Jackson (1895: 493) en el Index Kewensis, sinonimia que se mantiene en su versión en CD-ROM de 1997.

5. Orobanche schultzii Mutel, Fl. Franç. 2: 429 (1835)

Kopsia schultzii (Mutel) Bég.

Orobanche trichocalyx sensu Beck, non Webb \& Berthel. 207).

Ic.: Pujadas Salvà \& Lora González (1996:

Planta de 12-23 cm. Tallo de (3)4-6,5 mm de diámetro en su mitad, escasamente bulboso o apenas engrosado en la base $(4-13 \mathrm{~mm}$ de diámetro), simple o ramificado, pubescente glandular, blanquecino. Hojas de (5)9-15(17) $x(1,5) 2-4(6) \mathrm{mm}$, ovadas o lanceoladas. Inflorescencia de 6-12 x 3-3,7 cm, cilíndrica, con ápice ligeramente piramidal, subcomosa, densa a veces algo laxa en la base. Flores sésiles, las inferiores frecuentemente pediceladas, con pedicelos de hasta $9 \mathrm{~mm}$. Brácteas de (10)12-16(20) x (1,2)2-3(4) mm, estrechamente lanceoladas, a veces lineares, blanco cremosas. Bractéolas de $9-13$ x $(0,4) 0,7$ $1 \mathrm{~mm}$, linear lanceoladas, ápice filiforme. Cáliz de (10)13-16 mm, con dientes 1,5-3 veces la longitud del tubo (igualando la longitud del tubo de la corola), linear lanceolados con ápice filiforme, y un quinto diente adaxial de c.1 $\mathrm{mm}$, blanco cremoso. Corola de (14)17-21 mm, erecto patente, ligeramente curvada, hinchada en la base, estrechada en la inserción de los filamentos, de 1,5-2(3) $\mathrm{mm}$ de anchura, estrechamente tubular infundibuliforme, ligeramente ampliada en la garganta, de (3)4$6 \mathrm{~mm}$ de ancho, pubescente glandular, blanquecina, frecuentemente con limbo teñido de azul pálido. Labio superior, bilobado, inicialmente porrecto, posteriormente con lóbulos patentes, lóbulos elípticos, más o menos redondeados, acuminados, el inferior porrecto, con lóbulos iguales, elípticos, acuminados, repandos, de margen cortamente ciliado, con pelos glandulares de 0,1-0,2 mm. Filamentos insertos a (3)4-7 mm de la base de la corola, cortamente pubérulos en la base; anteras glabras. Ovario glabro. Estilo peloso glandular, blanco. Estigma blanco.

En matorrales degradados, a veces subruderalizados, de los pisos termo y mesomediterráneo, posiblemente sobre Thapsia villosa L. 100-1200 m. IV-V(VI).

\section{Material estudiado:}

ALMERIA: Puerto de Sta. María de Nieva, 30SWG8658, 900 m, 30-V-1994, A. Pujadas, COA 17465.

HUELVA: Huelva, in collibus vicinis, 2-V-1903, C. Pau, MA 114816.

JAÉN: Sa Mágina, Rastras de Albánchez, 1200 m, 19-VI-1926,Cuatrecasas, BC 46547 (sub Lathraea lavandulacea in Cuatrecasas, 1929: 429). Los Cañones. Los Villares, 12-VI-1991, A. Pujadas \& A. Lora, COA 17466.

MÁLAGA: Ctra. Málaga-Coín Km 13-14, Alhaurín de la Torre, 31-III-1994, M.R. Rubio, COA 17467. Alahurín de la Torre, junto al campo de Tiro, sobre Thapsia villosa L.?, 30-III-1995, A. Pujadas \& A. Lora, COA 17468. Sierra de Alhama, Cjo. de Marchamonas, en grietas con Parietaria officinalis 
L., calizas, 850-900 m, 6-VII-1973, B. Cabezudo \& B. Valdés, SEV 102940.

Nota: Además Beck (1930: 91) la señala para Alora y Ronda (Málaga) y en el Genil (Granada). La planta de Ronda citada por Beck (1930: 88) como O. trichocalyx y reconocida por Willkomm (1893: 190) debe de considerarse como O. schultzii. Valdés (1987: 557) indica la presencia de $O$. trichocalyx sensu Beck en Andalucía Occidental y aunque no hemos podido estudiar este material de referencia, creemos que se trata también de $O$. schultzii.

6. Orobanche arenaria Borkh., Neues Mag. Bot.: 6 (1794)

Orobanche laevis L.

Phelypaea arenaria (Borkh.) Walp.

Ic.: Beck (1930: 62, figs. B-L); Bonnier (1911-1935: pl. 463, fig. 2142 [sub Phelypaea arenaria]); Coste (1937: 64, fig. 2809 [sub Phelypaea arenaria]); Kreutz (1995: 33, 34).

Planta de (20)24-40(50) cm. Tallo de 3-9 $\mathrm{mm}$ de diámetro en su mitad, ligeramente o no engrosado en la base [5-12(20) $\mathrm{mm}$ de diámetro], simple, raramente ramificado, pubescente glandular, blanquecino. Hojas de (11)13-20 x 3-4 mm, lanceoladas. Inflorescencia de 9-20(26) x (2)3-4 cm, densa, de ápice redondeado. Flores sésiles, las basales a veces con pedicelos de 3-5(15) mm. Brácteas de $12-15(25) \times 3,5-5 \mathrm{~mm}$, ampliamente lanceoladas, blanquecinas, virando rápidamente a color pardo. Bractéolas de (9)10$15(18) \times 0,9-2 \mathrm{~mm}$, lineares o linear lanceoladas, blanquecinas, a veces ligeramente teñidas de púrpura. Cáliz de (13)16-21 mm, con dientes subulados, mayores o igualando su tubo, blanquecino, raramente teñido de púrpura. Corola de (18)21-30 mm, de erecta a erecto patente, ligeramente estrecha en la inserción de los filamentos, de $3-4 \mathrm{~mm}$ de ancho, y tubular, escasamente infundibuliforme en el ápice, de 0,9-1,4 mm de ancho en la garganta, pubescente glandular, azul violácea, blanquecina en la base. Labio superior cortamente bilobado, lóbulos generalmente patentes; el inferior con lóbulos obovados, obtusos, mucronados, ligeramente deflexos, de margen cortamente ciliado, con pelos glandulares de 0,1-0,2 mm. Filamentos insertos a 3,5-6 mm de la base de la corola, subglabros o glabrescentes; anteras pelosas en la base y sutura con pelos de 0,3-0,8 mm, blancas. Ovario peloso glandular en su ápice, a veces glabro. Estilo con pelos glandulares cortos, a veces esparcidos, blanco. Estigma blanco.

En matorrales xerofíticos degradados, subruderalizados, de los pisos termo a supramediterráneo, sobre Artemisia glutinosa DC. 100-1950 m. V-VII.

\section{Material estudiado:}

ALMERIA: Sierra entre Vélez Rubio y Sta. Ma . de Nieva, sobre Achillea millefolium (sic.), 8-VI-1951, Hno. Jerónimo, ALME 1723. Base del Maimón, 30SWG7970, $1100 \mathrm{~m}, 3-\mathrm{VI}-1987$, Blanca \& Cueto, ALME s/n. Colinas hacia Filabres, s/f, s/leg., BCF 3990 1. El Puertecico, Cruce a Los Cabrera de Abajo, talud de carretera, 30SWG8554, 850 m, 11-V-1994, A. Pujadas, COA 17330. Arroyo de El Saliente, Los Cerricos, Oria, 30SWG7254, 11-V-1994, A. Pujadas, COA 17337. Santuario El Saliente, sobre Artemisia glutinosa, 30SWG7453, $900 \mathrm{~m}, 16-\mathrm{V}-$ 1994, A. Pujadas, COA 17333. Calar Alto, 30SWG3819, 1950 m, 25-IV-1994, A. Pujadas, COA 17336. Lúcar, 30SWG5141, 950 m, 25-V-1994, A. Pujadas, COA 17338. Km 3 de Cantoria a Albánchez, sobre Artemisia glutinosa, 30SWG7453, 360 m, 25-V-1994, A. Pujadas, COA 17348. Puerto de Sta. María de Nieva, 30SWG8659, 1040 m, 30V-1994, A. Pujadas, COA 17343. Cruce de Los Tonosas a Vélez Rubio, sobre Artemisia glutinosa, 30SWG8663, 950 m, 30-V-1994, A. Pujadas, COA 17347. Km 1 de María a Vélez Blanco, 30SWG7474, 1100 m, 14-VI-1994, A. Pujadas, COA 17339. Aulago y cumbre de Sierra Filabres, 12-VII-1978, R. Sagredo, $\mathrm{H}^{\circ}$ LA SALLE s/n. Muela Montalbiche, 2-VII-1973, R. Sagredo, $\mathrm{H}^{\circ}$ LA SALLE s/n. Alcolea, 5-VI-1959, R. Sagredo, $\mathrm{H}^{\circ}$ LA SALLE s/n. Barranco Abrucena, 30-VII-1959, R. Sagredo, H $^{\circ}$ LA SALLE s/n. Camino de Vélez - De María a La Puebla - La 
Sagra, s/f, Clemente, MA 114831. Cañada de Clave, Vélez Rubio, sobre Artemisia, 18-V-1987, A. García Gea, MUB 40077.

GRANADA: Mecina Bombarón, Alpujarras, 29VI-1990, A. Pujadas \& P. Poyato, COA 17455. Cerca del río Trevélez, Trevélez, sobre Artemisia glutinosa, $1500 \mathrm{~m}, 30-\mathrm{VI}-1990$, A. Pujadas \& P. Poyato, COA 17335. Puebla de D. Fadrique a Santiago de la Espada, km 6, sobre Artemisia glutinosa, 19-VII-1999, A. Pujadas, COA 28906. Güejar Sierra, la vereda de La Estrella, 1100 m, sobre Artemisia glutinosa, 9-VI-2000, A. Pujadas \& E. Triano, COA 29506. Huéscar, $S^{a}$ de la Sagra, prox. Cortijo Ferrario, 1610 m, A.M. Negrillo, GDA 11938. Lanjarón, Cortijo del Olivo, VF5785, 600 m, 24-V-1979, J. Molero Mesa, GDA 11253, (sub O. ramosa subsp. mutelli in Molero-Mesa \& PérezRaya, 1987: 266). Sa de Baza, Rambla del Agua, WGO2, parasitando caméfitos del matorral heliófilo, 28-VI-1984, J. Torres et al., GDAC 27139. Sa de Baza, Cerro de la Pastora, WG23, parásito de caméfitos del matorral heliófilo, 4-VII-1984, J. Torres et al., GDAC 27138. El Fargue, lomas de Casablanca, parásita en un romeral (sic.), 28-VI1988, A. González et al., GDAC 32616. Sa de Baza, Cjo. del Pollo, 1600 m, 23-VII-1975, B. Cabezudo \& S. Talavera, SEV 102444. Huéscar, $S^{a}$ de Moncayo, calcareous soils, WG3395, 22-VI-1988, Valdés et al. SEV s/n. Almaciles, Campos de Bugejar, gypsophilous soils, WH6104, 1100 m, 27VI-1988, B. Valdés et al., n' 2461, SEV s/n (sub $O$. purpurea in Valdés \& Talavera, 1991: 204).

JAEN: Sa Mágina, 14-VII-1926, Cuatrecasas, MA 114832. Between Tobos and Ves, bed of river Zumeta, WH4523, $950 \mathrm{~m}, 25-\mathrm{VI}-1988, B$. Valdés et al., $\mathrm{n}^{\circ}$ 2206, SEV s/n (sub O. purpurea in Valdés \& Talavera, 1991: 204).

MÁLAGA: Cerca de Maro, Arroyo de la Miel, 25VII-1988, A. Pujadas, COA 13532. Nerja, Río de la Miel, sobre Artemisia glutinosa, 20-V-1989, A. Pujadas, COA 13888.

SEVILLA: Sevilla, s/f, J. Rodríguez, MA 114785.

Nota: La nomenclatura de esta especie corresponde a la propuesta por Turland \& Rumsey (1997). Beck (1930: 103) la cita en Sierra de Alibe (sic.) ¿Algibe?, basándose en una recolección de Porta y Rigo

7. Orobanche purpurea Jacq., Enum. Stirp. Vindob. 108, 252 (1762)

Phelypaea caerulea C.A. Mey.
Ic.: Bolòs \& Vigo (1996: 500); Kreutz (1995: 42, 43)[óptimo]; Pignatti (1982: 610).

Planta de 19-29 cm. Tallo delgado de 1,5$4 \mathrm{~mm}$ de diámetro en la mitad, débilmente bulboso en la base (3-7 mm de diámetro), simple, densamente pubescente glandular, teñido de azul púrpura. Hojas 9-11 x 2-3 mm, lanceoladas. Inflorescencia 5-9 x 2,9-4 cm, cilíndrica, ápice redondeado, algo laxa. Flores sésiles. Brácteas 8-12 × 2,5-4 mm, lanceoladas, teñidas de color purpúreo. Bractéolas 7-9 x 0,6-1,1 mm, lineares, ocroleucas hacia la base, purpúreas en el ápice. Cáliz 9-12 mm, con dientes mucho menores que su tubo, lanceolados, largamente acuminados, con un quinto diente adaxial desarrollado, ocroleuco hacia la base, azul púrpura en el ápice (aspecto pulverulento, en seco). Corola 18-21 mm, erecto patente a patente, apenas hinchada en la base, ligeramente estrechada en la inserción de los filamentos, de 2-3 mm de ancho, y tubular escasamente infundibuliforme, poco ensanchada en la garganta, de 5-6 mm de ancho, densamente pubescente glandular, base ocroleuca, limbo con las venas marcadas, teñidas de púrpura, a veces de azul pálido. Labio superior emarginado con los lóbulos patentes, el inferior con lóbulos iguales o subiguales, ovados, a veces cortamente apiculados, reflejos, margen cortamente ciliado con pelos glandulares de 0,1-0,3 mm. Filamentos insertos horizontalmente a $3-4 \mathrm{~mm}$ de la base de la corola, pelosos en la base, blancos de base amarilla; anteras vilosas, con pelos de hasta $0,6 \mathrm{~mm}$, en su base y a lo largo de la sutura. Ovario subglabro, con escasos pelos en su ápice. Estilo con pelos glandulares esparcidos, azul púrpura. Estigma blanco.

Sobre compuestas del género Achillea. Recolectada sobre Achillea odorata L., en herbazales inundados temporalmente, en el piso supramediterráneo. 1900-2000 m. VII-VIII. 
Material estudiado:

JAÉN. Sierra Mágina, Torres, El Almadén, sobre Achillea odorata L., 30SVG5377, 5-IX-1997, A. Pujadas \& M. Mazariegos, COA 25416. Sierra Mágina, El Almadén, sobre Achillea, 30SVG5376, 1940 m, 4-VII-1998, A. Pujadas \& L. Plaza, COA 25557.

Nota: Beck (1930: 107) no reconoce expresamente su presencia en la Península Ibérica, aunque incluye la sinonimia de Phelypaea coerulea con la referencia de Willkomm (1870: 627). Willkomm (1870: 628) la cita en la provincia de Córdoba, Pérez Lara (1889: 141) en Cádiz, en el Puerto de Santa María y en Gibraltar [citas que recoge Willkomm (1893: 189)] y Sagredo (1987: 420) en la provincia de Almería. Por otra parte, las citas de Valdés y Talavera (1991: 204) para las provincias de Granada y Málaga corresponden a $O$. arenaria, como se indica anteriormente. La referencia de Abu Sbaih \& Jury (1991: 42) para el sur de España, VF37, RNG 3350, es $O$. arenaria y la de Gibraltar, RNG 22, corresponde a $O$. mutelii.

AGRADECIMIENTOS. A los conservadores de los Herbarios: $\mathrm{ABH}, \mathrm{ALME}, \mathrm{BC}, \mathrm{BCC}, \mathrm{BCF}$, COLEGIO LA SALLE DE ALMERIA, COFC, GDA, GDAC, JACA, JAEN, MA, MAF, MGC, MPU, MUB, RNG y SEV por las facilidades en la consulta de sus respectivos herbarios. Al Dr. Aguedo Marrero por su aportación sobre Phelypaea trichocalyx de Canarias. Y a todos los colaboradores que han contribuído con sus recolecciones. A Laura Plaza por la revisión del manuscrito.

\section{BIBLIOGRAFÍA}

ABU SBAIH, H.A. \& S.L. JURY -1994- Seed micromorphology and taxonomy in Orobanche (Orobanchaceae). Fl. Medit. 4: 41-48.

BECK, G. -1890- Monographie der Gattung Orobanche. Bibliotheca Botanica 19: 1-275.

BECK, G. -1930- Orobanchaceae, in: Engler (Ed.), Das Pflanzanreich, 96 (IV-261), pp. 1-348. Berlin.
BOLÒS, O. \& J. VIGO -1996- Flora dels Països Catalans, vol. 3. Barcelona.

BONNIER, G. -1911/1935-Flore Complete Illustrée en couleurs de France, Suisse et Belgique. Tome VIII, fasc. 78. Paris.

BRUMMITT, R.K. \& C.E. POWELL (Eds.) -1992Authors of Plant Names. Kew.

CHATER, A. O. \& D.A. WEBB -1972-Orobanche, in: Tutin et al. (Eds.), Flora Europaea 3: 286293. Cambridge.

COSTE, H. -1937-Flore descriptive et illustrée de la France de la Corse et des contrés limitrophes. Vol. III. París.

CUATRECASAS, J. -1929- Estudios sobre la flora y vegetación del macizo de Mágina. Tab. Mus. Ci. Nat. Ser. Bot. 12: 3-510.

FERNÁNDEZ, C., Ma .C. CRUZ, M. LÓPEZPULIDO, C. AMEZCUA y D. CASADO -1991Flora de Andalucía. Catálogo bibliográfico de las plantas vasculares. Jaén.

FERNÁNDEZ LÓPEZ, C. -1983- Fuentes para la Flora de Jaén. Jaén.

FERNÁNDEZ LÓPEZ, C. \& P. MONTSERRAT RECODER -1985- Plantas vasculares de Jaén en el herbario JACA. Blancoana 3: 81-85.

GALIANO, E.F. \& V.H. HEYWOOD -1960Catálogo de plantas de la provincia de Jaén (Mitad Oriental). Jaén.

GREUTER, W.R., H.M. BURDET \& G. LONG 1989- Med-Checklist. Vol.4. (LauraceaeRhamnaceae). Genève.

GUIMARAES, J.A. -1903- Monografia das Orobanchaceas. Broteria: 1-188.

JACKSON, B.D. -1895-Index Kewensis. Vol. 2. Oxford

KREUTZ, C.A.J. -1995-Orobanche. The European broomrape species. Central and Northern Europe. Maastricht.

MOLERO-MESA, J. y F. PÉREZ-RAYA -1987La Flora de Sierra Nevada. Avance sobre el catálogo florístico nevadense. Granada.

MOLINO-OLMEDO, F., A. PUJADAS-SALVÀ, C. FERNÁNDEZ-GARCÍA-ROJO y C. FERNÁNDEZ-LÓPEZ -1995- Orobanche de Jaén hasta 1993. Blancoana 12: 47-50.

PÉREZ LARA, J.M. -1889- Flórula gaditana. Pars tertia. Anales Soc. Esp. Hist. Nat. 18: 35-143.

PIGNATTI, S. -1982- Flora d'Italia, Vol II. Bologna.

PUJADAS, A., Á. LORA y M.B. CRESPO -1997- 
Orobanche tunetana G. Beck (Orobanchaceae), especie nueva para el continente europeo. Anales Jard. Bot. Madrid 55(2): 277-283.

PUJADAS SALVÀ, A., E. HERNÁNDEZBERMEJO \& J.A.R. OLIVEIRA-VELLOSO 1994- The Genus Orobanche in Andalusia (southern Spain): Taxonomical, chorological \& ecological aspects. In: Pieterse, Verklej \& Ter Borg (Eds.), Biology and management of Orobanche: 132-137. Amsterdam.

PUJADAS SALVÀ, A. y A. LORA GONZÁLEZ 1996- El género Orobanche L. (Orobanchaceae) en la provincia de Almería, SE de España. Acta Bot. Malacitana 21: 199-220.

RUMSEY, F.J. \& S.L. JURY -1991- An account of Orobanche L. in Britain and Ireland. Watsonia 18: 257-295.

SAGREDO, R. -1987- Flora de Almería. Almería.

TALAVERA, S., C. AEDO, S. CASTROVIEJO, C. ROMEROZARCO, L. SÁEZ, F.J. SALGUEIRO y M. VELAYOS (Eds.) -1999- Flora Ibérica. Plantas vasculares de la Península Ibérica e Islas Baleares. Vol. VII(I). Leguminosae (partim). Madrid.

TURLAND, N. \& F. RUMSEY -1997- Proposals to reject the names Orobanche major and $O$. laevis (Orobanchaceae). Taxon 46: 787-791.

TUTIN, T.G., V.H. HEYWOOD, N.A. BURGES, D.M. MOORE, D.H. VALENTINE, S.M. WALTERS \& D.A. WEBB (Eds.) -1964/1980Flora Europaea, Vols. 1-5. Cambridge.

VALDÉS, B. -1987- Orobanche L., in Valdés et al. (Eds.), Flora Vascular de Andalucía Occidental, Vol. 2: 550-557. Barcelona.

VALDÉS, B. \& S. TALAVERA -1991- Check-list of the vascular plants collected during Iter Mediterraneum I. Bocconea 1: 43-286.

VALDÉS, B., S. TALAVERA y E. FERNÁNDEZGALIANO (Eds.) -1987- Flora Vascular de Andalucía Occidental, Vols. 1-3. Barcelona.
WEBB, P.B. \& S. BERTHELOT -1845- Histoire naturelle des Iles Canaries: 3(2.3), livr. 68. Paris.

WEBB, P.B. \& S. BERTHELOT -1847- Histoire naturelle des Iles Canaries: 3(2.3), livr. 92. Paris.

WILLKOMM, H.M. -1870- Orobanchaceae, in: Willkomm \& Lange (Eds.), Prodromus Florae Hispanicae 2: 620-632. Stuttgart.

WILLKOMM, H.M. -1893-Supplementum prodromi florae hispanicae. Stuttgart.

Aceptado para su publicación en julio de 2000

Dirección: Departamento de Ciencias y Recursos Agrícolas y Forestales. Universidad de Córdoba. Apdo. 3048. E-14080 Córdoba. E-mail: cr1pusaa@uco.es 